\title{
A Ship Driving Teaching System Based on Multi-level Virtual Reality Technology
}

\author{
https://doi.org/10.3991/ijet.v11i11.6249 \\ Feng-hua Cao \\ Inner Mongolia University of Finance and Economics, Inner Mongolia, China
}

\begin{abstract}
The present ship driving teaching system cannot ensure that all seafarers or students have enough time to go into training for basic skills, and it is not practical or feasible in all cases. We construct a consistent VR marine training environment to enhance maritime education and training at all levels. The basic equation of key algorithm involving the multilevel virtual reality ship driving teaching system is presented in this paper. The system is equipped with V.Dragon 2000. The application results show that the system can project visual system with wide field of view, and instructor control station etc. The training environment provides a strong visual reinforcement and a strong sense of immersion.
\end{abstract}

Index Terms-ship driving teaching system, multi-level virtual reality, marine simulation system

\section{INTRODUCTION}

Since the most effective approach to training is to "learn by doing", Maritime education and training depend on enough practice to foster qualified and competent personnel working in the maritime industry both afloat and ashore. While we know a full functional marine simulator is a very effective method available not only because they can simulate the operational characteristics of many types of ships, but also because they may replicate real world situations[1-2]. We also recognize that this is not practical or feasible in all cases, since the limited accessibility (time or field), immobility, long development cycle, limited application life, expensive development cost and system maintenance of the full mission marine simulator, it cannot ensure that all seafarers or students have enough time to go into training for basic skills. In addition, while the universities thrive on improved enrollment, they face the physical limitations of learning environment for curriculum practice [3-5].

To solve the Problem, based on the finished distributed marine simulation system and the newly developed desktop multi-functional marine teaching system, now Dalian Maritime University (DMU) starts developing a webbased marine training environment (WBMTE) fully combining web technology, virtual environment, e-learning tools and high fidelity simulation software to create a new training medium for maritime education and training [6]. The distributed marine simulation system, the desktop multi-functional marine teaching system and the webbased marine training environment supplement each other to make a multi-level training system in the whole marine training process. This paper will present how multi-level training will complementary each other to construct a consistent VR marine training environment to enhance maritime education and training at all levels.
Marine simulator is a valuable and effective medium for maritime education and training and is becoming widespread to foster the qualified and competent personnel for working in the maritime industry. However, many problems with most marine simulation systems today limit their pervasive use; the limited accessibility, immobility, the high expenditure, the long development cycle, and the difficult maintenance etc. A web-based maritime training environment is proposed in this paper as another marine training level, which fully combining web technology, VR, high fidelity simulation, and e-learning tools to create a more cost-effective and flexible training environment still with very realistic and high-interactive simulation characteristics [7]. The distributed marine simulation system, desktop Multi-functional Marine Teaching System and the proposed web-based maritime training environment are combined together into a multi-level training system, which will provide a consistent and more effective training medium for improving maritime education and training.

The distributed marine simulation system is composed of instructor control station, primary own-ship and multi auxiliary own ships without number limitation. All parts is connected by Ethernet and all own-ships can be $\mathrm{M}$ in sight of one another" through radar image and visual system. A large screen projected realistic visual system with wide field of view and a real scale bridge with integrated equipment's generating a realistic training environment. Figure 1 is a snapshot of the primary Own-Ship Bridge.

The desktop multi-functional marine teaching system is a networked PC-based integrated teaching/ training environment (see fig. 2), which aims at supporting collaborative teaching/ training in both asynchronous and synchronous modes among teachers and students on campus. The system is scalable and consists of many unit multimedia classrooms, which is composed of one teacher platform



Figure 1. The own-ship bridge of marine simulator 




Figure 2. The mutational marine teaching lab(left) and the multifunctional marine teaching unit (right)

and thirty student platforms. Both platforms are equipped with desktop engine and rudder steering console, multimedia network card, PC and other multimedia facilities. Moreover teacher platform is furnished with networked multimedia teaching switchboard, large screen projector, printer and etc. for facilitating multimedia instruction [89].

\section{OVERVIEW}

With the development of virtual reality and humancomputer interaction, more and more people pay attention to virtual driving simulator. Interactive technology in the virtual driving simulator becomes the hot and difficult topic of virtual reality and human-computer interaction field. Traditional virtual driving game with the mouse, keyboard and other equipment complete the interaction. The operating mode is boring. In order to achieve the virtual driving simulation system with strong authenticity, the high efficient of interaction and advanced interaction with good immersion, the real vehicle driving steering wheel, brakes and other interactive devices are adopted in this system.

As a comprehensive integration technology, virtual reality technology is involved in the human-computer interaction technology, computer graphics technology, display technology, sensor technology and artificial intelligence. The application area is relatively wide, and it depends on the computer technology, which is a kind of high technical simulation system. From the point of view of narrow sense, virtual reality technology can be defined as follows. Through the computer technology, the real feeling of seeing and hearing is realized closely to human beings. The users can experience the virtual design by specific audio-visual device. When moving the location, such as users connected to the computer auxiliary equipment can be user displacement sensor, and related operations, which affect transmission through 3D to the user the use of equipment, so that the user can real feel design environment. From the Angle of the generalized, virtual reality technology can be defined as that the related environment or things exist in the world, its existence in the form of physical or functional significance, can be implemented through the actual operation, such as cyberspace, etc.

The software and hardware technology are two important factors of virtual reality technology and the hardware technology includes computer and related basic devices. At present, among the computer input and output devices, the development of visual and audio equipment are relatively perfect, but the smell and taste of the hardware device is still in research. According to the characteristics of the virtual reality technology, virtual reality technology can be divided into two dimensional model of virtual and panorama virtual methods. According to the degree of immersion and interaction mode, the virtual reality technology can be divided into distributed VR system, desktop VR system, the superposition type VR system and immersive VR system. In the virtual system, since the desktop virtual reality system does not need to use large server and processor, the cost of the desktop is lowest. The design of virtual scene can be realized by personal computers.

A Web-based marine training environment (WBMTE) aims to make full fidelity simulations, currently resident only on the DMS or DMMTS mentioned above, accessible to a much broader users via a desktop or laptop computer over the Internet or Internet network. It use the full fidelity simulations as the basis combined with synthetic virtual training environments for familiarization, procedures and decision-making training [10]. The WBMTE provide an attractive, easily navigated browser-based training interface integrating Web, VR technology, marine simulation, and e-learning methods and technologies.

We have developed a prototype system by applying WBMTE technologies, architecture and concepts to training some co-pilot/ seafarer. The prototype system includes: 1) A full ship handling simulation software simulation the ship's maneuvering motion through different water area, including graphical representation of the ship handling device with 2D instrumentation, run-time information during ship navigation and $3 \mathrm{D}$ navigation environment with a virtual ship. An instructor operator station and scenario generator is researched. 
The prototype of the WBMT environment offers many basic services: providing information, accessing to WBMT web-site, presentation of marine course material, tutorials, marine skill training, sharing knowledge/skills facility (chatting room), etc. In our case, the WBMT site interface layout is shown in figure 3 . Users' first login is necessary to enter the marine training page below. The next is to set the training environment information including the fog, wind, current the daytime and ship type etc.

Figure 4 shows the interface provided by a web browser, where the 3D VRML training world and the ship handling interface created by one Java applet are embedded into a HTML page. The VRML world is a viewer showing the $3 \mathrm{D}$ vivid navigation environment. A controlling panel performs most interactions between the user and the virtual ship for ease of use. Communication between the VRML world and the controller is achieved by the VRML External Authoring Interface (EAI), which provides a set of methods that an external application can use to interact with, and dynamically update a 3D scene in real-time. Thus, the rudder or engine operations are mapped from the controller widgets to the virtual ship in the viewer through a Java Applet.

Both DMMTS and WBMTE utilize the same high fidelity simulation as DMS to provide real-time updated images or videos created by networked PCs and construct a visual virtual training environment for maritime education and training, such as Radar plotting/ ARP A operation ship maneuvering, collision avoidance and etc. Thus protecting investments and ensuring consistent training is obtained.

The major differences are that the DMMTS system makes use of desktop simulated steering console to replicate the functionality of various parts of real scale bridge equipment and controls of DMS While the WBMTE use mouse keyboard and emulated soft panels to substitute for the simulated steering console. The DMS provide a large screen projected visual training environment with wide field of view, while the DMMTS and the WBMTE only generate a virtual training scene on pc screen. So the immersion feeling of the WBMTE and the DMMTS is worse than the DMS, and the visual quality of the WBMTE is lower than that of the other two for the geometry replication through the limited network bandwidth. The DMMTS is portable and flexible for more users* training than the DMSt but the DMS is larger in scale and immersing for more effective and complex navigation training. The WBMTE have the benefit to provide simulation-based elearning to more anytime and anywhere. How three training complement each other to ensure more efficient performance will be demonstrated in detail in the following part.

\section{METHOD AND ALGORITHM}

In recent years, Virtual Reality (VR) technology has been paid extensive attention in computer field. Virtual reality technology is designing a lifelike virtual environment based on the computer technology, which can access interaction between the user and the environment by use of the essential device. It is an essential condition to design a lifelike model for user feeling the lifelike virtual environment. 3D modeling technology is the foundation of the VR system and the key step and technology of the application. It is an important part in virtual reality system when developing a virtual reality system.



Figure 3. The marine teaching simulation web site



Figure 4. The multifunctional marine teaching unit

$\mathrm{Hu}$ [11] developed the virtual reality system of the digital launch site in Creator and Vega environment, which realized the digitalization of launch site, real-time simulation of Satellite Launch and assistant of Satellite launch mission. The paper carried out study at aspect of the complicated modeling, the visual scene designing, the realtime optimizing and the system establishing to realize some important conceptions and methods of VR modeling. On the basis of the theory study of VR modeling and characteristic of system the paper choose geometry-based modeling and rendering scheme to create the digital space launch site with the outstanding 3D modeling and presented software of Vega in the field of visual scene simulation. For solving the contradiction between real-time and reality, the paper researched the key modeling technology, and then the paper proposed the effective approaches for realtime improvement from the aspect of structure optimizing and modeling optimizing. The last part firstly discussed the Vega software and the rending elements, and then presented the actualizing scheme of the digital space launch site system with the high scene complexity, realtime and interaction. The system can display scene, walk through, drive the motion model and display the special effect, and so on. The specialization of the digital space launch site and visual flight simulation system makes the thesis possess the representativeness of Virtual Reality modeling.

According to the degree of immersion and interaction mode, the virtual reality technology can be divided into distributed VR system, desktop VR system, the superposition type VR system and immersive VR system. In the virtual system, since the desktop virtual reality 
system does not need to use large server and processor, the cost of the desktop is lowest.

The design of virtual scene can be realized by personal computers. All parts is connected by Ethernet and all ownships can be $\mathrm{M}$ in sight of one another" through radar image and visual system. A large screen projected realistic visual system with wide field of view and a real scale bridge with integrated equipment's generating a realistic training environment.

For pedagogical reason, this phase means that students should have a basic knowledge of the navigation curriculum and related topics before they start the simulation training by accessing Web-based training courses or take the instruction in multimedia class mentioned above (see figure 2). Instructors and students interact using video, text, slide and sound combined to create powerful, immediate impressions of theoretical background material and guidance for learning and practices,

Then after learning the basic navigation theory, students would use multiple marine simulation and teaching software to conduct navigation training under the instructor's guidance, which further improves students* deep understanding in practice.

In class training time, students start training by steering the rudder and engine, operating other simulated panels, interacting with real-time updated graphical displays and communicating with instructor or other students synchronously to acquire a much deeper knowledge about the safety navigation theory and principles taught. Group-to group collaboration training (such as collision avoidance) in a virtual training environment greatly enhances students * learning experience and accelerates greater cognitive development. Figure 3 shows a 2D ECDIS interface and $3 \mathrm{D}$ synthetic navigation scene. Figure 5 is the radar simulation interface and navigation information interface. All those tools assistant the safely navigation training conveniently, the whole training process can be controlled by the teacher platform: setup, pause, resume or stop for some medial explanation or instruction. Students' training process can be easily recorded or grasped by capturing visual or numerical dates of overall training status. Finally, the results of training will be replayed or printed for discussion analysis and evaluation according to some complex evaluation metrics for special training task. Then students can be given a standard performance rating for the procedure performed. Radar simulation interface, own ship navigation information and ECDIS are showed in Fig. 5. Training result shows that students were actively engaged by this type of interactive visual training experience and gained a good knowledge of navigation theory and technology.

The basic equation of key algorithm is shown as the equation (1):

$$
(N, s k) \leftarrow \operatorname{Key}\left(1^{k}\right)
$$

This formula is used to generate file checksum parameter which is denoted by:

$$
\begin{aligned}
& r \leftarrow\{0,1\}^{k} ; s k \leftarrow\{e, d, r\} ; \\
& \text { Output }\{N, s k\} ;
\end{aligned}
$$

The Euler function is:

$$
\phi(N)=(p-1)(q-1)
$$

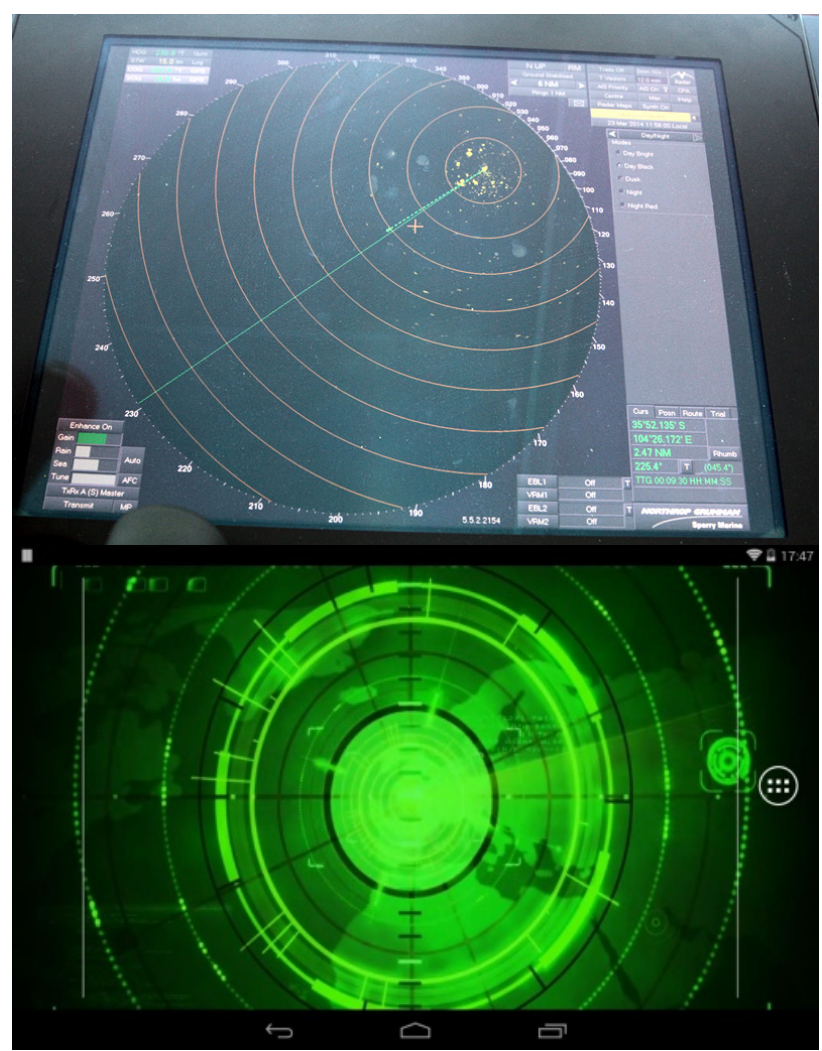

Figure 5. The radar simulation interface

Then choose an integer e to satisfy the following equation 4:

$$
\left\{\begin{array}{l}
1<e<\phi(N) \\
\operatorname{gcd}(e, \phi(N))=1
\end{array}\right.
$$

Then finally export $(\mathrm{N}, \mathrm{sk})$ in Tag algorithm, we can get the optimization equation (5):

$$
\left(T_{0}, T_{2}, \ldots T_{n-1}\right) \leftarrow \operatorname{Tag}(p k, s k, m)
$$

The formula generates labels for each file block.

$$
\begin{gathered}
\operatorname{for}(j=0 ; j \leq n-1 ; j++) ; \\
\left\{W_{j}=r^{*}(j+1) ; T_{i}\right. \\
\left.=\left[h\left(W_{j}\right)^{*} m_{j}\right]^{c} \bmod N\right\} ; \\
\text { Output }\left(T_{0}, T_{2}, \ldots T_{n-1}\right) ;
\end{gathered}
$$

And local fractional integral of $f(x)$ defined by Eq.9.

$$
\begin{aligned}
& { }_{a} I_{b}^{(\alpha)} f(t)=\frac{1}{\Gamma(1+\alpha)} \int_{a}^{b} f(t)(d t)^{\alpha} \\
& =\frac{1}{\Gamma(1+\alpha)} \lim _{\Delta t \rightarrow 0} \sum_{j=0}^{j=N-1} f\left(t_{\mathrm{j}}\right)\left(\Delta t_{\mathrm{j}}\right)^{\alpha}
\end{aligned}
$$

Its local fractional Hilbert transform, denoted by $f_{x}^{H, \alpha}(x)$ is defined by

$$
\begin{aligned}
& H_{\alpha}\{f(t)\}=\hat{f}_{H}^{\alpha}(x) \\
& =\frac{1}{\Gamma(1+\alpha)} \int_{R} \frac{f(t)}{(t-x)^{\alpha}}(d t)^{\alpha}
\end{aligned}
$$


Where $x$ is real and the integral is treated as a Canchy principal value, that is,

$$
\begin{aligned}
& \frac{1}{\Gamma(1+\alpha)} \int_{R} \frac{f(t)}{(t-x)^{\alpha}}(d t)^{\alpha} \\
& =\lim _{\varepsilon \rightarrow 0}\left[\frac{1}{\Gamma(1+\alpha)} \int_{-\infty}^{x-\varepsilon} \frac{f(t)}{(t-x)^{\alpha}}(d t)^{\alpha}+\right. \\
& \left.\frac{1}{\Gamma(1+\alpha)} \int_{x+\varepsilon}^{\infty} \frac{f(t)}{(t-x)^{\alpha}}(d t)^{\alpha}\right]
\end{aligned}
$$

To obtain the inverse local fractional Hilbert transform, write again Eq. (11) as

$$
\begin{aligned}
& \hat{f}_{H}^{\alpha}(x)=\frac{1}{\Gamma(1+\alpha)} \int_{-\infty}^{\infty} \frac{f(t)}{(t-x)^{\alpha}}(d t)^{\alpha} \\
& =\frac{1}{\Gamma(1+\alpha)} \int_{-\infty}^{\infty} f(t) g(x-t)(d t)^{\alpha} \\
& =f(x) * g(x),
\end{aligned}
$$

The equation of motion is as follows:

$$
\begin{gathered}
\partial_{j}\left(C_{i j k l} \partial_{k} u_{l}+e_{k i j} \partial_{k} \varphi\right)-\rho \ddot{u}_{i}=0 \\
\Delta b_{j}=\left(y(k)-y_{m}(k)\right) w_{j} h_{j}\left(\frac{\left\|X-C_{j}\right\|^{2}}{b_{j}^{3}}\right) \\
b_{j}(k)=b_{j}(k-1)+\eta \Delta b_{j} \\
+\alpha\left(b_{j}(k-1)-b_{j}(k-2)\right) \\
\Delta c_{j, i}=\left(y(k)-y_{m}(k)\right) w_{j} \frac{x_{j}-c_{j, i}}{b_{j}^{2}}
\end{gathered}
$$

Under the linear theory, that is:

$$
\partial_{j}\left(e_{i j k l} \partial_{k} u_{l}-\eta_{k i j} \partial_{k} \varphi\right)=0
$$

\section{EXPERIMENT RESULT}

For further maximizing the effectiveness of training, the distributed marine simulation system can be used exclusively for advanced complex training or qualification training after the primary procedural training using desktop teaching system. V.Dragon 2000 is equipped with real scale bridge and relative instruments, large screen projected visual system with wide field of view, and instructor control station etc. So this training environment provides a strong visual reinforcement and a strong sense of immersion. Moreover, this training environment integrates visual, auditory and tactile training which allowing students to physically interact with the simulated real-ship equipment, watch and analyze visual navigation situation, listen to the 3D sound generated by motors or navigation environment, conduct some training interactively with other students to complete complex training mission. This audiovisual and tactile training environment creates a high interactive positive atmosphere, stimulates student's interests, aids retention and causes a wide range of both physiological

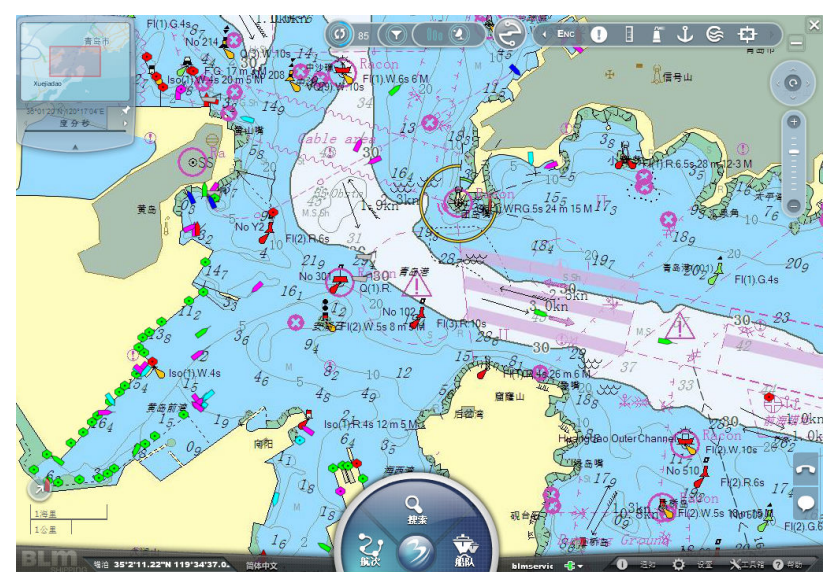

Figure 6. The navigation simulation interface

and psychological learning accelerators. Finally, a qualitative leap in improving maritime education and training is achieved. The training result and technical progress of students can be evaluated effectively by some method. Figure 6 shows the vivid viewing of the simulated navigation environment (from the left to right, they are the Sydney harbor in daytime and in night, the Singapore harbor and the Dalian harbor respectively).

\section{DISCUSSION}

The web-based marine training philosophy is to provide consistent training to students as they progress through basic, advanced, qualification and continuation training Before reaching classes, students can access web-based training courses for initial familiarization training. When instruction begins, the instructor would use WBMTE to conduct free-play simulations. These simulations would teach procedures for a particular ship handling training. After class instruction, students would use the WBMTE to conduct the free-play simulations on their own time and their own controlled pace. Students and teacher can communicate asynchronously via email, news group, ftp file transfer, shared discussing-board or message board freely and synchronously via text-based network chatting, networked telephone, interactive group discussion. During type-specific qualification training, WBMTE would be used in conjunction with DMSs and DMMTSs, which would ensure the student made efficient use of the simulator time for continuation training, transition training and mission training. WBMTE use the same simulation software as the full fidelity DMSs and DMMTSs, thus giving the student a consistent training experience and earn higher learning value throughout the overall training curriculum.

\section{CONCLUSION}

The DMS and DMMTSs have been employed in fifteen scientific or educational institutions in China and exported to Malaysia and Singapore. The widespread use and all users' immediate feedback demonstrate the effectiveness of enhancing seafarers or students' competency. Figure 1 show the DMS installed in JiMei University, NingBo University, Tianjin University of Technology and other scientific or educational institutions. In figure 2 shows a multimedia classrooms used for the maritime education and training in NingBo University is shown. Fig. 3 is a unit system of our multifunctional marine teaching system used by DaLian Ocean Shipping company. 
While school and technology leaders are employing DMSs and DMMTSs to increase academic achievement through enhancing students' skill and competency. They need to take Web-based training as the next level for the benefits of ability to train anytime and anywhere, wider accessibility, more interaction and resource share, more self-paced instruction, and more efficient maintenance. A web-based maritime training environment is proposed in this paper as another marine training level, which fully combining web technology, VR, high fidelity simulation, and e-learning tools to create a more cost-effective and flexible training environment still with very realistic and high-interactive simulation characteristics.

The distributed marine simulation system, desktop Multi-functional Marine Teaching System and the proposed web-based maritime training environment are combined together into a multi-level training system, which will provide a consistent and more effective training medium for improving maritime education and training. Furthermore, Existing simulations from high fidelity simulators are re-used, thus protecting investments and ensuring consistent training. A Web-based marine training environment (WBMTE) aims to make full fidelity simulations, currently resident only on the DMS or DMMTS mentioned above, accessible to a much broader users via a desktop or laptop computer over the Internet or Internet network. It use the full fidelity simulations as the basis combined with synthetic virtual training environments for familiarization, procedures and decision-making training.

Learning value is higher because WBMTE incorporates simulation into it, which immerses the student in the proper context while training and dramatically increases retention levels and learning effectiveness. Delivery of contentrich simulation to the PC or laptop - WBMTE - will achieve high rates of student proficiency and competency at a lower relative cost. Although WBMTE' s visual quality, immersion feeling is not as good as the large scale marine simulation system, this training paradigm can supplement the existing marine training system to improve marine education and training more efficiently. Our future work is to improve the prototype of WBMTE as an applied tool for maritime education and training.

\section{REFERENCES}

[1] H. Jing, "The Study on the Impact of Data Storage from Accounting Information Processing Procedure," International Journal of Database Theory and Application, vol. 8, no.3, pp. 323-332, June 2015. https://doi.org/10.14257/ijdta.2015.8.3.28

[2] Y. Zhou, "Application of Automatic Choreography Software Based on Virtual Technology in the Gymnastics Teaching," International Journal of Emerging Technologies in Learning, vol. 11, no.5, pp. 39-44, May 2016. https://doi.org/10.3991/ ijet.v11i05.5692

[3] D. Jiang, X. Ying, Y. Han, et al., "Collaborative Multi-hop Routing in Cognitive Wireless Networks," Wireless Personal Communications, vol. 86, no.2, pp. 901-923, January 2016. https://doi.org/10.1007/s11277-015-2961-6

[4] Z. R. Ren, S. Ni, H. Li, et al, "Development and Application of the Virtual Vessel Experiment Teaching System," Ship \& Ocean Engineering, 2014.

[5] H. Jing, "Node deployment algorithm based on perception model of wireless sensor network," International Journal of Automation Technology,vol.9, no.3, pp. 210-215, April 2015. https://doi.org/10.20965/ijat.2015.p0210

[6] D. Amoako-Sakyi, H. Amonoo-Kuofi, "Problem-based learning in resource-poor settings: lessons from a medical school in Ghana," Bmc Medical Education, vol.15, no.1, pp. 1-8, April 2015. https://doi.org/10.1186/s12909-015-0501-4

[7] R. Q. Zhu, S. L. Chen, Y. Ni, et al., "Study and Practice on Teaching Model of Ship Fluid Mechanics Based on Network Course Platform," Ship \& Ocean Engineering, 2014.

[8] J. Lu, F. X. Zhu, X.F. Wen, et al., "Study on the Teaching Quality Assurance System for Postgraduate of Marine Engineering Specialty," Ship \& Ocean Engineering, 2014.

[9] X. Yang, D. Li. "On the Sailing Vocational Students' Simulation Ship Driving and Turbine Management Measures," Guide of Science \& Education, 2014.

[10] L. Zhang, D.Wang, "Research on the Ship Antifouling Experiment Based on Oil Spill Emergency Simulation System," Science Education Article Collects, 2014.

X. Hu, "Research on Small-class Teaching Based on Virtual Engine Room Desktop System in Higher Vocational Colleges," Ship \& Ocean Engineering, 2014.

\section{AUTHOR}

Feng-hua Cao is with Inner Mongolia University of Finance and Economics, Inner Mongolia, China (e-mail: cjdx_cfh@163.com).

Submitted 09 September 2016. Published as resubmitted by the authors 16 October 2016. 